\title{
Rationale, Design, and Baseline Characteristics of ARTS-DN: A Randomized Study to Assess the Safety and Efficacy of Finerenone in Patients with Type 2 Diabetes Mellitus and a Clinical Diagnosis of Diabetic Nephropathy
}

\author{
Luis M. Ruilope $^{\mathrm{a}}$ Rajiv Agarwal ${ }^{\mathrm{b}}$ Juliana C. Chan ${ }^{\mathrm{c}}$ Mark E. Cooper ${ }^{\mathrm{d}}$ \\ Ron T. Gansevoort ${ }^{\mathrm{e}}$ Hermann Haller ${ }^{f}{\text { Giuseppe Remuzzik, I }{ }^{\mathrm{k}} \text { Peter Rossing }}^{\mathrm{m}}$ \\ Roland E. Schmiederg Christina Nowack ${ }^{\mathrm{h}} \quad$ Anna C. Ferreira $^{\mathrm{n}} \quad$ Alexander Pieper $^{\mathrm{i}}$ \\ Nina Kimmeskamp-Kirschbaum ${ }^{j}$ George L. Bakris ${ }^{\circ}$ \\ ${ }^{a}$ Institute of Investigation and Hypertension Unit, Hospital 12 de Octubre, Madrid, Spain; ${ }^{b}$ Richard L. Roudebush \\ VA Medical Center, Indianapolis, Ind., USA; ' Department of Medicine and Therapeutics, The Chinese University of \\ Hong Kong, Hong Kong, SAR, China; ${ }^{\mathrm{d} B a k e r}$ IDI Heart and Diabetes Institute, Melbourne, Australia; ${ }^{e}$ Department \\ of Nephrology, University Medical Center Groningen, Groningen, The Netherlands; ${ }^{\mathrm{f}}$ Departments of Nephrology \\ and Hypertension, Hannover Medical School, Hannover, ${ }^{9}$ Department of Nephrology and Hypertension, University \\ Hospital Erlangen, Erlangen, ${ }^{\mathrm{h}}$ Global Clinical Development, Bayer HealthCare AG, Leverkusen, ${ }^{i}$ M.A.R.C.O. GmbH \& \\ Co. KG, Düsseldorf, and jGlobal Research and Development Statistics, Bayer HealthCare AG, Leverkusen, Germany; \\ kIRCCS - Istituto di Ricerche Farmacologiche Mario Negri, Clinical Research Center for Rare Diseases 'Aldo e Cele \\ Daccò', Ranica (Bergamo), and 'Unit of Nephrology and Dialysis, Azienda Ospedaliera Papa Giovanni XXIII, Bergamo, \\ Italy; ${ }^{\mathrm{m} S t e n o}$ Diabetes Center, Gentofte, Denmark; ${ }^{\mathrm{n}}$ Global Clinical Development, Bayer S.A., Bayer HealthCare, São \\ Paulo, Brazil; ${ }^{\circ}$ The University of Chicago Medicine, Chicago, Ill., USA
}

\section{Key Words}

Antagonist · Diabetic nephropathy $\cdot$ Heart failure .

Mineralocorticoid receptor · Type 2 diabetes mellitus

\section{Abstract \\ Background/Aims: Finerenone decreases albuminuria in patients having heart failure with reduced ejection fraction and mild-to-moderate (stage 2-3) chronic kidney disease. The MinerAlocorticoid Receptor Antagonist Tolerability Study-Diabetic Nephropathy (ARTS-DN; NCT01874431) is a multicenter, randomized, double-blind, placebo-controlled, parallel-group, phase $2 \mathrm{~b}$ study. ARTS-DN investigated}

whether the mineralocorticoid receptor antagonist finerenone reduces albuminuria without causing major alterations in serum potassium levels in patients with type 2 diabetes mellitus and a clinical diagnosis of DN who were receiving a renin-angiotensin-system (RAS) inhibitor. Methods: Patients were randomized to oral finerenone $1.25-20 \mathrm{mg}$ or placebo once daily. The primary objectives were to assess the ratio of the urinary albumin-to-creatinine ratio at day 90 to that at baseline in patients receiving finerenone, and to compare it with that in the placebo group. Additional exploratory analyses included evaluating changes from baseline in serum potassium levels, efficacy and safety biomarkers, and healthrelated quality of life. Results: Of 1,501 patients screened,

\section{KARGER}

E-Mail karger@karger.com www.karger.com/ajn
(C) 2015 S. Karger AG, Basel

0250-8095/15/0406-0572\$39.50/0
Prof. Luis M. Ruilope

Institute of Investigation and Hypertension Unit

Hospital 12 de Octubre

ES-28041 Madrid (Spain)

E-Mail ruilope@ad-hocbox.com 
821 (the sample population) received at least one dose of finerenone/placebo. Baseline characteristics included: male, 77.8\%; white, $84.2 \%$; very high albuminuria (formerly macroalbuminuria), 38.4\%; high albuminuria (formerly microalbuminuria), 60.3\%; median (range) estimated glomerular filtration rate, $66.3(24.5-130.7) \mathrm{ml} / \mathrm{min} / 1.73 \mathrm{~m}^{2}$; and systolic blood pressure (mean \pm standard deviation), $138.1 \pm 14.4$ $\mathrm{mm} \mathrm{Hg}$. There was a history of cardiovascular disease in $39.6 \%$, diabetic neuropathy in $20.0 \%$, and diabetic retinopathy in $19.9 \%$ of patients. Conclusion: ARTS-DN is the first phase $2 \mathrm{~b}$ trial of finerenone in combination with a RAS inhibitor in patients with type 2 diabetes mellitus and a clinical diagnosis of DN.

(c) 2015 S. Karger AG, Basel

\section{Introduction}

Diabetic kidney disease (DKD) is the leading cause of end-stage renal disease (ESRD) in the western world [1]. Among patients with type 2 diabetes mellitus, high (formerly known as micro-) albuminuria - a marker of cardiovascular risk [2] - has a global prevalence of approximately $40 \%[3,4]$. In post hoc analyses of large randomized trials in patients with diabetes, decreases in albuminuria are associated not only with a slower progression to ESRD in the long term but also with a reduction in the incidence of cardiovascular (CV) outcomes and mortality $[5,6]$. Consequently, reduction of albuminuria is considered to be a marker of slowed nephropathy progression in the treatment of chronic kidney disease (CKD) [7].

For the medical management of patients with $\mathrm{DKD}$, reduction of albuminuria has become a primary aim, in addition to optimizing control of hyperglycemia and blood pressure [8]. Guidelines currently recommend treatment with angiotensin-converting enzyme inhibitors (ACEIs) or angiotensin II receptor blockers (ARBs) for diabetic patients with hypertension and very high (formerly known as macro-) or high albuminuria [8]. Both ACEIs and ARBs act via inhibition of the renin-angiotensin-system (RAS) [9-11]. Despite initial down-regulation of plasma aldosterone levels, up to $50 \%$ of patients treated with a RAS blocker experience elevations of the hormone within a year of initiating treatment [12-14]. This 'aldosterone breakthrough/escape' is associated with increases in albuminuria and impairment of kidney function $[13,15,16]$.

In patients with $\mathrm{CKD}$, systematic reviews of small studies have suggested that, even when added to ACEIs and $\mathrm{ARBs}$, mineralocorticoid receptor (MR) antagonists

Finerenone for Type 2 Diabetes Mellitus and Diabetic Nephropathy
(MRAs) substantially reduce proteinuria $[17,18]$. Thus, among adults with DKD, a potential role for the steroidal MRAs spironolactone and eplerenone as antiproteinuric agents has been identified in explorative clinical studies [19-22]. Furthermore, MRAs are well known for their ability to reduce mortality and morbidity in patients with heart failure with reduced ejection fraction (HFrEF) [2325]. As there is a high risk of CV-associated mortality and morbidity in patients with $\mathrm{DKD}$, it is possible that MRAs may also have a part to play in preventing CV events in this population. However, long-term prospective studies confirming whether MRAs slow progression to ESRD in patients with DKD are lacking.

Finerenone (BAY 94-8862) is a next-generation oral MRA with a non-steroidal chemical structure [26]. In vitro, finerenone has shown higher selectivity for the MR over other steroid hormone receptors compared with spironolactone and improved affinity for the MR compared with eplerenone [26]. At equi-natriuretic doses in recent preclinical studies, finerenone reduced proteinuria and cardiac hypertrophy more efficiently than eplerenone [27]. In the phase 2a MinerAlocorticoid Receptor Antagonist Tolerability Study (ARTS), encouraging safety and efficacy profiles were observed in patients with HFrEF and mild-to-moderate CKD treated with finerenone [28]. Importantly, finerenone doses of $2.5-10 \mathrm{mg} /$ day reduced albuminuria from baseline, particularly in patients with elevated albuminuria. In addition, finerenone was associated with a lower increase in serum potassium concentration and incidence of hyperkalemia than spironolactone. Thus, finerenone may be able to address the unmet medical need of safely managing albuminuria in patients with type 2 diabetes mellitus and a clinical diagnosis of DKD.

The ARTS-Diabetic Nephropathy (ARTS-DN; ClinicalTrials.gov identifier: NCT01874431), which is now completed, was designed to compare the short-term efficacy and safety of different once-daily oral doses of finerenone and placebo in patients with type 2 diabetes mellitus and DN who are receiving an ACEI or an ARB. Here we describe the ARTS-DN design and the baseline characteristics of the study population.

\section{Patients and Methods}

ARTS-DN is a multicenter, randomized, double-blind, placebocontrolled, parallel-group, phase $2 \mathrm{~b}$ study aiming to compare the effects of finerenone $1.25-20 \mathrm{mg}$ once-daily with placebo on top of standard of care in adults with type 2 diabetes mellitus and DN.

Following a run-in and screening period of up to 12 weeks, eligible patients were randomized to once-daily finerenone $1.25-$ 


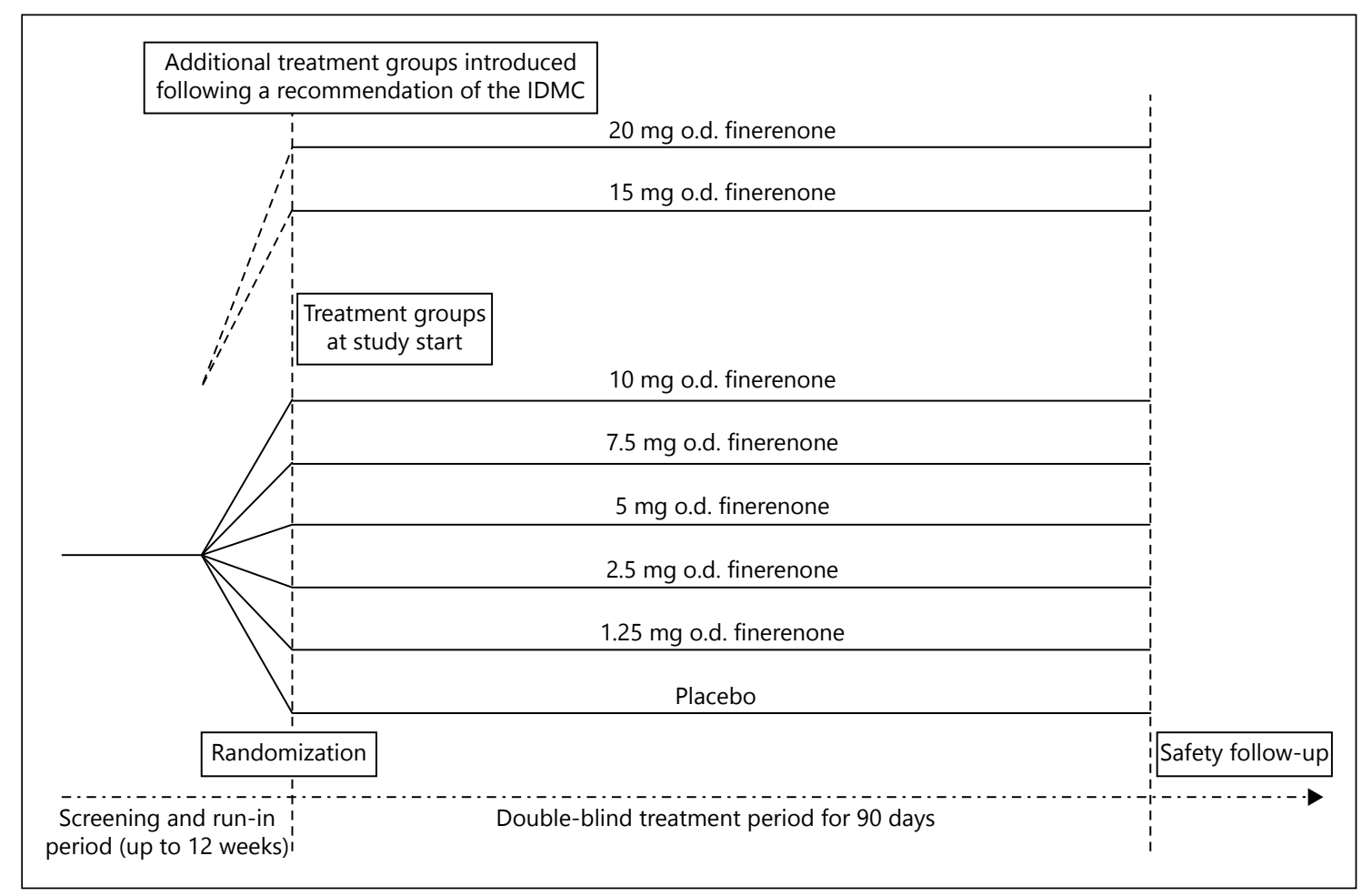

Fig. 1. Study flowchart. IDMC = Independent data monitoring committee; o.d. = once daily.

$10 \mathrm{mg}$ or placebo in combination with the current standard of care for 90 days (fig. 1). Treatment arms of finerenone 15 and $20 \mathrm{mg}$ once-daily were added on the recommendation of an independent data monitoring committee (IDMC) after review of the safety data from the ongoing study.

\section{Patients}

After a run-in visit, patients who fulfilled the eligibility criteria (table 1) were enrolled in a run-in period of up to 12 weeks. To be included, patients had to have type 2 diabetes (i.e. medical history of a 2 -hour plasma glucose level $\geq 11.1 \mathrm{mmol} / 1$ during an oral glucose tolerance test or of a fasting glucose level $\geq 7.0 \mathrm{mmol} / \mathrm{l}$; or glycated hemoglobin $\left[\mathrm{HbA}_{1 \mathrm{c}}\right]$ levels of at least $6.5 \%$ in the medical history or at the run-in visit; or be receiving treatment with oral antidiabetics and/or insulin). Treatment with at least the minimum recommended dose of an ACEI and/or an $\mathrm{ARB}$ prior to the run-in period was also a requirement for inclusion in the study (following the run-in period, only an ACEI or an ARB, but not both, was allowed); antihypertensive therapy was optimized for renal and cardiovascular disease protection, according to local guidelines. As part of the run-in period, a screening visit to confirm eligibility for randomization took place within 14 days of the planned randomization. At this visit, it was assessed whether the patient still met the eligibility criteria (table 1) while receiving at least the minimum recommended dose of an ACEI or ARB. Adults meeting the above criteria for having type 2 diabetes mellitus who had a clinical diagnosis of $\mathrm{DN}$ (i.e. consistent urinary albumin-to-creatinine ratios [UACRs] in two of three first morning samples, with both being $\geq 300 \mathrm{mg} / \mathrm{g}$ [ $\geq 34 \mathrm{mg} / \mathrm{mmol}]$ or both being $\geq 30$ to $<300 \mathrm{mg} / \mathrm{g}$ [ $\geq 3.4$ to $<34 \mathrm{mg} /$ mmol] plus an estimated glomerular filtration rate [eGFR] $\geq 30$ to $<90 \mathrm{ml} / \mathrm{min} / 1.73 \mathrm{~m}^{2}$ ) and a serum potassium level of $4.8 \mathrm{mmol} / \mathrm{l}$ or less at the run-in and screening visits were randomized to treatment (table 1). Patients with an eGFR of $30-45 \mathrm{ml} / \mathrm{min} / 1.73 \mathrm{~m}^{2}$ at the runin visit had to start treatment with a non-potassium sparing-diuretic if not already receiving such treatment; this treatment must have been stable for at least 4 weeks before the screening visit (table 1).

Patients with a clinical diagnosis of HFrEF and persistent symptoms (New York Heart Association class II-IV) at the run-in visit, or $\mathrm{HbA}_{1 \mathrm{c}}$ levels of more than $12 \%$, a UACR of more than $3,000 \mathrm{mg} / \mathrm{g}$ or hypertension at the run-in or screening visits were excluded from the study (table 1). Hypertension was defined at the run-in visit as mean sitting systolic blood pressure of at least 180 $\mathrm{mm} \mathrm{Hg}$ or mean sitting diastolic blood pressure of at least $110 \mathrm{~mm}$ $\mathrm{Hg}$, and at the screening visit as mean sitting systolic blood pressure of at least $160 \mathrm{~mm} \mathrm{Hg}$ or mean sitting diastolic blood pressure of at least $100 \mathrm{~mm} \mathrm{Hg}$. If blood pressure was considered by the investigator to be uncontrolled during the double-blind study period, non-potassium-sparing diuretics were the first-line treatment if not already being used. Thereafter, antihypertensive medications could be added to the treatment regimen according to local guidelines. If the blood pressure was still not considered to be controlled by the investigator, the patient was withdrawn from the study.

Patients with non-diabetic renal disease, known bilateral clinically relevant renal artery stenosis or Addison's disease, or who experienced a stroke, transient ischemic cerebral attack, acute coronary syndrome, or hospitalization for worsening HF within 30 days before the run-in visit were not eligible for participation in the study. 
Table 1. Main inclusion and exclusion criteria of ARTS-DN

Main inclusion criteria

Men or women $\geq 18$ years (women with childbearing potential were required to have a negative pregnancy test and to have agreed to use adequate contraception)

Patients with type 2 diabetes mellitus fulfilling at least one of the following criteria:

Receiving treatment with oral antidiabetics and/or insulin

Medical history of fasting glucose $\geq 7.0 \mathrm{mmol} / \mathrm{l}$

Medical history of 2-hour plasma glucose $\geq 11.1 \mathrm{mmol} / 1$

during an oral glucose tolerance test

$\mathrm{HbA}_{1 \mathrm{c}} \geq 6.5 \%$ (NGSP/DCCT) in the medical history or at the run-in visit A clinical diagnosis of $\mathrm{DN}$ based on at least one of the following criteria at the run-in/screening visit:

Persistent very high albuminuria: UACR of $\geq 300 \mathrm{mg} / \mathrm{g}$ ( $\geq 34 \mathrm{mg} /$ $\mathrm{mmol}$ ) in two of three first morning samples and estimated eGFR (CKD-EPI) $\geq 30$ to $<90 \mathrm{ml} / \mathrm{min} / 1.73 \mathrm{~m}^{2}$

Persistent high albuminuria: UACR of $\geq 30$ to $<300 \mathrm{mg} / \mathrm{g}$ ( $\geq 3.4$ to $<34 \mathrm{mg} / \mathrm{mmol}$ ) in two of three first morning samples and eGFR $(\mathrm{CKD}-\mathrm{EPI}) \geq 30$ to $<90 \mathrm{ml} / \mathrm{min} / 1.73 \mathrm{~m}^{2}$

In patients with an eGFR (CKD-EPI) of $30-45 \mathrm{ml} / \mathrm{min} / 1.73 \mathrm{~m}^{2}$, treatment with a non-potassium-sparing diuretic at the screening visit and without any adjustments for $\geq 4$ weeks beforehand

Treatment with at least the minimum recommended dose of an ACEI and/or ARB for at least 3 months without any adjustments to this therapy for at least 4 weeks prior to the screening visit Serum potassium $\leq 4.8 \mathrm{mmol} / \mathrm{l}$ at both the run-in and the screening visit
Main exclusion criteria

Non-diabetic renal disease

Known bilateral clinically relevant renal artery stenosis $(>75 \%$ reduction in artery diameter)

$\mathrm{HbA}_{1 \mathrm{c}}>12 \%$ at the run-in or screening visit

UACR $>3,000 \mathrm{mg} / \mathrm{g}(339 \mathrm{mg} / \mathrm{mmol})$ in any of the first morning samples at the run-in or screening visit Hypertension with mean sitting SBP $\geq 180 \mathrm{~mm} \mathrm{Hg}$ or mean sitting $\mathrm{DBP} \geq 110 \mathrm{~mm} \mathrm{Hg}$ at the run-in visit, or with mean sitting SBP $\geq 160 \mathrm{~mm}$ Hg or mean sitting DBP $\geq 100 \mathrm{~mm} \mathrm{Hg}$ at the screening visit

Clinical diagnosis of HFrEF and persistent symptoms (NYHA class II-IV) at the run-in visit, or stroke, transient ischemic cerebral attack, acute coronary syndrome, or hospitalization for worsening HF within 30 days before the run-in visit

Concomitant therapy with eplerenone, spironolactone or any renin inhibitor or potassium-sparing diuretic that cannot be discontinued for the run-in and the treatment period

Concomitant therapy with high-dose ( $>500 \mathrm{mg} /$ day) acetylsalicylic acid or daily treatment with other nonsteroidal anti-inflammatory drugs for more than 5 consecutive days

Use of potent CYP3A4 inhibitors/inducers or strong CYP2C8 inhibitors

ACEI = Angiotensin-converting enzyme inhibitor; ARB = angiotensin II receptor blocker; CKD-EPI = Chronic Kidney Disease Epidemiology Collaboration; DBP = diastolic blood pressure; DCCT = Diabetes Control and Complications Trial; DN = diabetic nephropathy; $\mathrm{eGFR}=$ estimated glomerular filtration rate; $\mathrm{HbA}_{1 \mathrm{c}}=$ glycated hemoglobin; $\mathrm{HF}=$ heart failure; $\mathrm{HFrEF}=\mathrm{HF}$ with reduced ejection fraction; NGSP = National Glycohemoglobin Standardization Program; NYHA = New York Heart Association; SBP = systolic blood pressure; UACR = urinary albumin-to-creatinine ratio.

\section{Objectives}

The primary efficacy variable was the ratio of the UACR at visit 5 (day $90 \pm 2$ ) to the UACR at baseline (visit 1; day 1 ). The main objective of ARTS-DN was to determine the effect of treatment with different doses of finerenone or placebo on this efficacy variable.

Further exploratory objectives were to assess:

- the ratio of UACR at visit 3 (day $30 \pm 2$ ) and visit 4 (day $60 \pm$ 2) to UACR at baseline;

- the incidence of regression of albuminuria from baseline to visit 5, with regression of albuminuria defined as a change from either very high albuminuria (UACR $\geq 300 \mathrm{mg} / \mathrm{g}$ ) to high albuminuria (UACR $\geq 30$ to $<300 \mathrm{mg} / \mathrm{g}$ )/normal albuminuria (UACR $<30 \mathrm{mg} / \mathrm{g}$ ) or from high albuminuria to normal albuminuria, in all cases accompanied by a change in UACR of more than $30 \%$;

- changes in levels of efficacy biomarkers (aldosterone, B-type natriuretic peptide [BNP], N-terminal proBNP [NT-proBNP], galectin-3) from baseline to visits 3, 4 and 5;

- changes in health-related quality of life (HRQoL) from baseline to visit 3 , visit 5 , and the follow-up visit, using the Kidney Dis- ease Quality of Life-36 and EuroQol 5-Dimension, 3-Level questionnaires.

A safety profile will be established using data from physical examinations, blood pressure and heart rate monitoring, electrocardiograms, adverse event monitoring, and blood sample analysis (hematology and clinical chemistry, including measurements of levels of liver enzymes, creatinine, potassium, $\mathrm{HbA}_{1 \mathrm{c}}$, and safety biomarkers [troponin T and cystatin C]). The IDMC assessed safety and tolerability, with a particular focus on changes in serum potassium (number of patients with hyperkalemia [a confirmed value of serum potassium $\geq 5.6 \mathrm{mmol} / \mathrm{l}]$ ) and eGFR (number of patients with an eGFR decrease of $\geq 30 \%$ from baseline).

\section{Study Medication}

Finerenone was administered as oral, immediate-release tablets. Eligible patients were randomized 1:1:1:1:1:1 in the 14 days following a screening visit to receive once-daily finerenone at doses of $1.25,2.5,5.0,7.5$, or $10 \mathrm{mg}$ or matching placebo for 90 days. Two additional treatment arms, finerenone 15 and $20 \mathrm{mg}$ once daily, were added on the recommendation of the IDMC in December 2013. When the additional treatment arms were added, 
randomization was adapted to obtain equally balanced sample sizes across all treatment groups at the end of the study. Randomization was stratified by region (Europe, North America, Asia, others [Australia, Israel, South Africa]) and type of albuminuria (very high albuminuria or high albuminuria at screening).

Ideally, any medical therapy (e.g. antidiabetic, antihypertensive, or statin) would not be changed between the screening visit and the last dose of study drug; however, if this was necessary, the patient could continue to receive the study drug. Concomitant medications that were not allowed during the study included any marketed aldosterone antagonist or renin inhibitor; high-dose acetylsalicylic acid ( $>500 \mathrm{mg} /$ day); daily treatment with non-steroidal anti-inflammatory agents; potent cytochrome P450 (CYP) isoenzyme 3A4 inhibitors or inducers; and strong CYP2C8 inhibitors such as gemfibrozil (table 1).

\section{Serum/Plasma Potassium Monitoring}

Patients had to maintain their normal diet throughout the study and were not given any specific advice on dietary sodium or potassium restrictions. With the exception of non-potassiumsparing diuretics, potassium-lowering agents (e.g. sodium polystyrene sulfonate, calcium polystyrene sulfonate, insulin and glucose infusion) were not allowed to be started during treatment with study drug. In the case of hyperkalemia occurring with study treatment, the study treatment was discontinued prior to starting a potassium-lowering agent. Any potassium supplementation was stopped prior to randomization if potassium levels were within the normal range. If potassium levels were low at randomization or at any of the following visits, potassium supplementation was continued or re-started until potassium values were within the normal range again.

If a patient underwent a change in clinical status that was known to influence serum/plasma electrolyte levels or fluid balance (e.g. vomiting and/or diarrhea for $>1$ day), it was recommended that serum/plasma potassium concentration be reassessed as soon as possible after the acute event. Any reassessment of serum/plasma potassium concentration had to be analyzed locally and centrally. At each scheduled visit, blood samples were collected for the measurement of parameters including serum/ plasma potassium concentration. Investigators were instructed to stop the study drug permanently in the event of a confirmed potassium concentration of $5.6 \mathrm{mmol} / \mathrm{l}$ or more or a locally measured potassium concentration of more than $6.0 \mathrm{mmol} / \mathrm{l}$. Such a discontinuation had to be reported to the sponsor within $24 \mathrm{~h}$ as a serious adverse event.

\section{Investigations}

Patients were assessed at the run-in visit, the screening visit, visit 1 (day 1 ), visit 2 (day $7 \pm 2$ ), visit 3 (day $30 \pm 2$ ), visit 4 (day $60 \pm 2$ ) and visit 5 (day $90 \pm 2$ ); details of the assessments made at each visit are given in the online supplemental data (for all online suppl. material, see www.karger.com/doi/10.1159/000371497).

Follow-up visits were scheduled for $30 \pm 5$ days after the last intake of study medication. Patients who discontinued the study prematurely were also assessed as soon as possible after discontinuation.

Urine samples taken from the first void in the morning at the patient's home on three consecutive days were used to measure the levels of urinary creatinine, albumin, sodium, and potassium. At visits 1 and 5, urine samples were taken before the first and last intake of study drug, respectively. A local dipstick test was used to confirm sample validity for central analysis. Urine samples were frozen at $-20^{\circ} \mathrm{C}$, apart from the sample for assessment of albuminuria, which remained at ambient temperature.

Blood samples were used to determine levels of efficacy biomarkers (BNP, NT-proBNP, galectin-3, and aldosterone), safety biomarkers (ultrasensitive troponin $\mathrm{T}$ and cystatin $\mathrm{C}$ ), and $\mathrm{HbA}_{1 \mathrm{c}}$, and for hematology, clinical chemistry, pharmacokinetic analysis, and assessment of iohexol plasma clearance (for eGFR estimations in a subset of the study population), as indicated in supplementary table. Serum samples were frozen at $-20^{\circ} \mathrm{C}$, apart from the samples for troponin $\mathrm{T}$ and aldosterone, which remained at ambient temperature. Plasma was frozen at $-20^{\circ} \mathrm{C}$, with the exception of samples for pharmacokinetic analysis, which were frozen at $-15^{\circ} \mathrm{C}$. Whole blood samples remained at ambient temperature.

\section{Statistics}

The following sets will be used for statistical analysis: the safety analysis set (SAF; all randomized patients who have taken at least one dose of study drug and for whom there are post-treatment data); the full analysis set (FAS; all patients in the SAF who have baseline and at least one post-baseline UACR value); and the perprotocol analysis set (PPS; all patients in the FAS who have a valid UACR value at visit 5 and no major protocol deviations).

Analysis of the primary efficacy variable will be performed in the FAS (primary analysis) and PPS (supportive analysis). Dosedependency will be assessed by fitting an analysis of covariance model (ANCOVA) to the log-transformed ratios of UACR at visit 5 to UACR at baseline, including the factors 'treatment group', 'region', and 'type of albuminuria' and the log-transformed baseline UACR as a covariate nested within 'type of albuminuria', and testing a pre-specified linear contrast $\left(\mathrm{L}_{8}{ }^{\prime}=[6.125,5.125,4.125\right.$, $2.125,0.125,-1.875,-5.875,-9.875]$, one-sided; significance level, $5 \%)$. Subsequent hierarchical pairwise comparisons to placebo will also be performed in case the primary hypothesis could be rejected.

In the primary analysis, a last observation carried forward method will be applied, whereby the higher UACR value from the premature discontinuation measurement and the follow-up measurement will be used to impute missing UACR values at visit 5 . Sensitivity analyses will also be conducted. In addition, descriptive statistics of the primary efficacy variable will be generated.

Further exploratory efficacy variables will be analyzed in the FAS and PPS. The ratios of UACRs at visits 3,4 , and 5 to those at baseline will be assessed by fitting a mixed-effect repeated measures model to the log-transformed ratios, with the same factors as for the primary analysis plus the factor time and the interaction between treatment and time. Changes in the type of albuminuria and categories of relative decreases of eGFR will be analyzed with frequency tables. Separate ANCOVAs, including factors of 'treatment group', 'region', and 'type of albuminuria', and with the baseline value as a covariate will be used to study changes in efficacy biomarkers and HRQoL scores from baseline to visits 3, 4, and 5 or visits 3 and 5, respectively.

Safety data will be assessed in the SAF. Adverse events and laboratory data will be analyzed by frequency tables and summary statistics, respectively. Changes in safety biomarkers, eGFR and levels of creatinine and serum potassium from baseline to visits 3, 4 , and 5 will be analyzed by separate ANCOVAs as for the efficacy biomarkers. The incidence of patients with a potassium value of at least $5.6 \mathrm{mmol} / \mathrm{l}$ or of greater than $6 \mathrm{mmol} / \mathrm{l}$, or in different catego- 
ries of eGFR decreases or categories of creatinine increases, will be investigated with frequency tables. Percentages of patients in the above categories in the finerenone treatment groups will be compared with those in the placebo group by chi-squared tests with continuity correction.

The ratio of UACR at visit 5 to UACR at baseline of 0.91 or 0.95 is assumed for placebo, whereas UACR ratios are expected to decrease with an increasing dose of finerenone until a ratio of 0.64 to 0.46 for finerenone $15 \mathrm{mg}$ in different scenarios. Sample size calculations were performed with nQuery Advisor ${ }^{\circledR} 7.0$ (Statistical Solutions, Cork, Ireland). A sample size of 75 patients who were valid for the FAS in each treatment group would give power of at least $83 \%$ to demonstrate a dose-dependent effect on the primary variable for seven treatment groups (doses up to $15 \mathrm{mg}$ ) using the linear contrast $\mathrm{L}_{7}{ }^{\prime}=(4.714,3.714,2.714,0.716,-1.286,-3.286$, -7.286 ) at a significance level of 0.05 (one-sided), assuming a common standard deviation of 1.25 on the log scale and a true contrast of the log-transformed UACR ratios of at least 3.937. It is expected that the power will increase in the case of eight treatment groups (doses up to $20 \mathrm{mg}$ ). Taking into account that the 15 and $20 \mathrm{mg}$ finerenone treatment arms were added, 600 patients were required in total. To achieve this, approximately 1,340 patients had to be enrolled into ARTS-DN (assuming a screening failure rate of up to $50 \%$ ) and approximately 670 had to be randomized among treatment groups (assuming a drop-out rate of $10 \%$ ). It was planned to increase the sample size when less than $35 \%$ of randomized patients were diagnosed with very high albuminuria. As a result, more than 670 patients were actually randomized.

\section{Ethics}

The procedures set out in this protocol pertaining to the conduct, evaluation, and documentation of this study were designed to ensure that the sponsor and investigator abide by Good Clinical Practice guidelines and follow the guiding principles detailed in the Declaration of Helsinki.

The study was carried out in keeping with applicable local law(s) and regulation(s). Documented approval from appropriate independent ethics committee(s) or institutional review board(s) was obtained for all participating centers/countries before the start of the study. All individuals gave their informed consent for participation.

\section{Results}

\section{Overall Cohort}

The study started in June 2013 and was clinically completed in August 2014. Of 1,501 patients screened at 148 sites, 823 patients were randomized and reviewed by medical experts (Australia, $\mathrm{n}=17$; Austria, $\mathrm{n}=27$; Bulgaria, $\mathrm{n}=$ 84; Canada, $\mathrm{n}=38$; Czech Republic, $\mathrm{n}=27$; Denmark, $\mathrm{n}=$ 71; Finland, $\mathrm{n}=52$; France, $\mathrm{n}=14$; Germany, $\mathrm{n}=14$; Hong Kong, $\mathrm{n}=7$; Hungary, $\mathrm{n}=25$; Israel, $\mathrm{n}=64$; Italy, $\mathrm{n}=82$; Netherlands, $\mathrm{n}=22$; Norway, $\mathrm{n}=7$; Poland, $\mathrm{n}=23$; Portugal, $\mathrm{n}=9$; Republic of Korea, $\mathrm{n}=12$; South Africa, $\mathrm{n}=51$ Spain, $\mathrm{n}=67$; Sweden, $\mathrm{n}=45$; Taiwan, $\mathrm{n}=22$; USA, $\mathrm{n}=43$ ). Of
Table 2. Demographics and baseline characteristics of all patients who were randomized to and recorded starting study treatment

\begin{tabular}{|c|c|}
\hline Characteristic & Total $(n=821)$ \\
\hline Men, $\mathrm{n}$ & $639(77.8)$ \\
\hline Age, years & $64.2 \pm 9.2$ \\
\hline \multicolumn{2}{|l|}{ Ethnicity, $\mathrm{n}$} \\
\hline Not hispanic/latino & $797(97.1)$ \\
\hline Hispanic/latino & $18(2.2)$ \\
\hline Not reported & $6(0.7)$ \\
\hline \multicolumn{2}{|l|}{ Race, $n$} \\
\hline White & $691(84.2)$ \\
\hline Black & $28(3.4)$ \\
\hline Asian & $84(10.2)$ \\
\hline Mixed & $16(1.9)$ \\
\hline Not reported & $2(0.2)$ \\
\hline BMI, $\mathrm{kg} / \mathrm{m}^{2}$ & $31.8 \pm 5.5$ \\
\hline \multicolumn{2}{|l|}{ Blood pressure, $\mathrm{mm} \mathrm{Hg}$} \\
\hline Systolic & $138.1 \pm 14.4$ \\
\hline Diastolic & $77.1 \pm 9.7$ \\
\hline \multicolumn{2}{|l|}{ Baseline laboratory variables } \\
\hline $\mathrm{UACR}, \mathrm{mg} / \mathrm{g}$ & $192.8[6.3-4,948.0]$ \\
\hline$>300 \mathrm{mg} / \mathrm{g}, \mathrm{n}$ & $301(36.7)$ \\
\hline$\geq 30$ to $<300 \mathrm{mg} / \mathrm{g}, \mathrm{n}$ & $498(60.7)$ \\
\hline$<30 \mathrm{mg} / \mathrm{g}, \mathrm{n}$ & $22(2.7)$ \\
\hline Serum potassium, $\mathrm{mmol} / \mathrm{l}$ & $4.29 \pm 0.42$ \\
\hline eGFR (CKD-EPI), $\mathrm{ml} / \mathrm{min} / 1.73 \mathrm{~m}^{2}$ & $66.3[24.5-130.7]$ \\
\hline$<30 \mathrm{ml} / \mathrm{min} / 1.73 \mathrm{~m}^{2}, \mathrm{n}$ & $16(1.9)$ \\
\hline $30-45 \mathrm{ml} / \mathrm{min} / 1.73 \mathrm{~m}^{2}, \mathrm{n}$ & $138(16.8)$ \\
\hline$>45-60 \mathrm{ml} / \mathrm{min} / 1.73 \mathrm{~m}^{2}, \mathrm{n}$ & $175(21.3)$ \\
\hline$>60 \mathrm{ml} / \mathrm{min} / 1.73 \mathrm{~m}^{2}, \mathrm{n}$ & $492(59.9)$ \\
\hline Serum creatinine (range), $\mathrm{mg} / \mathrm{dl}$ & $1.1[0.5-2.6]$ \\
\hline $\mathrm{BNP}, \mathrm{pg} / \mathrm{ml}$ & $43.0[2.5-2,060.5]$ \\
\hline NT-proBNP, pg/ml & $116.1[10.0-8,212.3]$ \\
\hline Galectin-3, ng/ml & $9.0[1.9-160.0]$ \\
\hline \multicolumn{2}{|l|}{ Troponin $\mathrm{T}, \mathrm{ng} / \mathrm{ml}$} \\
\hline$<0.05, \mathrm{n}$ & $795(96.8)$ \\
\hline $0.05-0.16, \mathrm{n}$ & $5(0.6)$ \\
\hline Not reported, $\mathrm{n}$ & $21(2.6)$ \\
\hline Cystatin C, ng/ml & $960.0[100.0-2,720.0]$ \\
\hline $\mathrm{HbA}_{1 c}, \%$ & $7.4[4.9-11.9]$ \\
\hline
\end{tabular}

Data are expressed as means $\pm \mathrm{SD}$, numbers with percentages in parentheses or medians with ranges in square brackets. $\mathrm{BMI}=$ Body mass index; $\mathrm{BNP}=\mathrm{B}$-type natriuretic peptide; $\mathrm{CKD}$-EPI = Chronic Kidney Disease Epidemiology Collaboration; eGFR = estimated glomerular filtration rate; $\mathrm{HbA}_{1 \mathrm{c}}=$ glycated hemoglobin; $\mathrm{NT}$-proBNP $=\mathrm{N}$-terminal proBNP; SD = standard deviation; UACR = urinary albumin-to-creatinine ratio. Data on BNP, NTproBNP, galectin-3 and cystatin $C$ were available for 804, 739, 807, and 812 patients, respectively.

these patients, two did not receive treatment: one owing to a protocol deviation and another because of withdrawal of informed consent. The baseline characteristics of the 821 patients who received at least one dose of finerenone/placebo (the sample population) are summarized in table 2 . 
Table 3. Medications of interest that were initiated before starting study treatment and diabetic disease and cardiovascular history of all patients who were randomized to and recorded starting study treatment

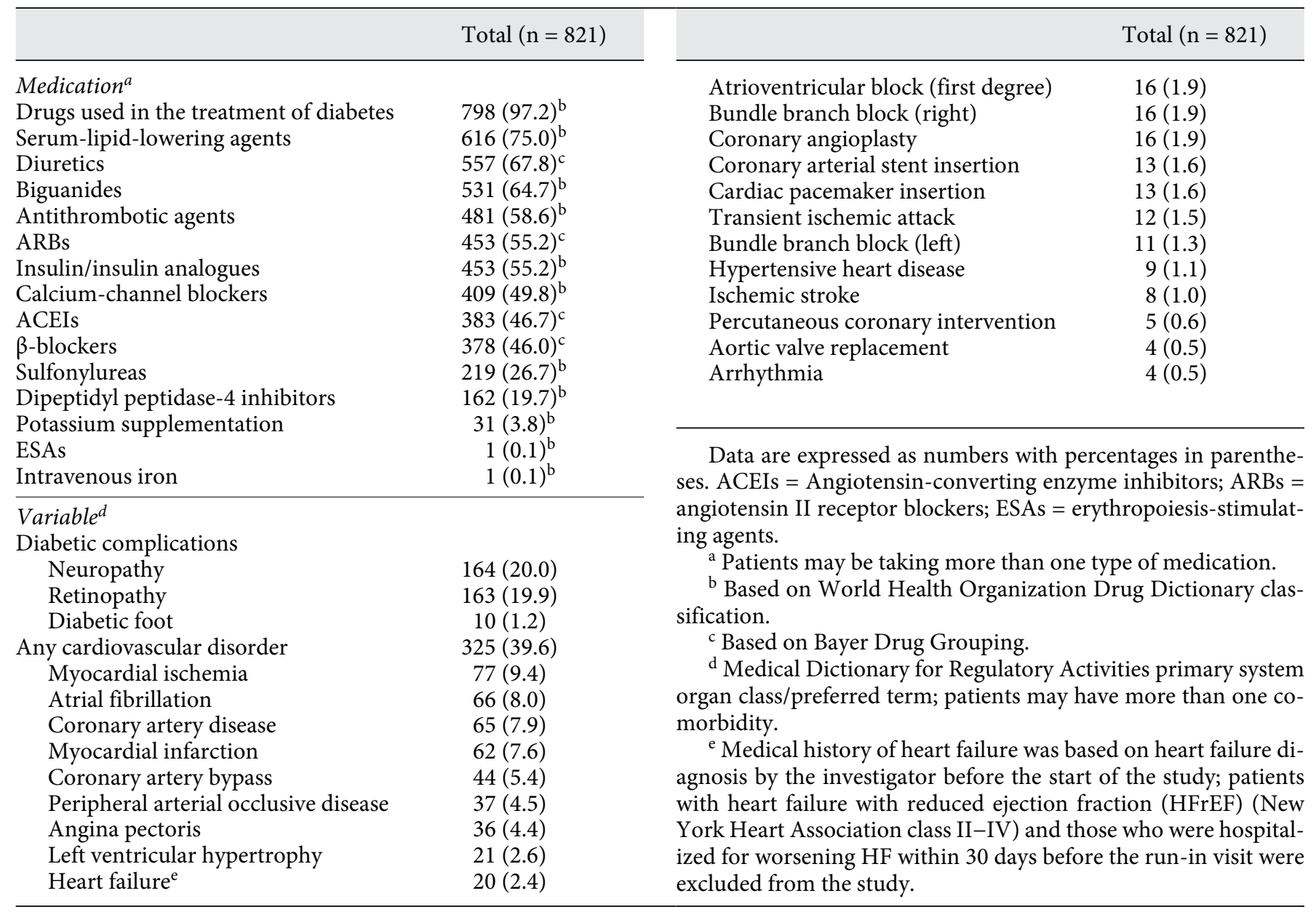

The majority of treated patients were men (77.8\%), and most were white (84.2\%); 69.1\% were European. At screening, UACR data were available for 815 patients: 495 $(60.3 \%)$ had high albuminuria; 315 (38.4\%) had very high albuminuria and $5(0.6 \%)$ had normal albuminuria.

At baseline, the median UACR was $192.8 \mathrm{mg} / \mathrm{g}$. In total, $60.7 \%$ of patients had high albuminuria, $36.7 \%$ had very high albuminuria, and $2.7 \%$ had normal albuminuria. The median eGFR was $66.3 \mathrm{ml} / \mathrm{min} / 1.73 \mathrm{~m}^{2}$, and $18.8 \%$ of treated patients had an eGFR less than or equal to $45 \mathrm{ml} / \mathrm{min} / 1.73 \mathrm{~m}^{2}$.

\section{Concomitant Medication and Medical History}

Medications initiated before baseline (including those stopped before baseline) are listed in table 3. ARBs and ACEIs were initiated prior to baseline in 55.2 and $46.7 \%$ of the study population, respectively, and calci- um-channel blockers in $49.8 \%$. In addition, $97.2 \%$ of patients were using medication to manage their diabetes, $75.0 \%$ were using agents to reduce lipid levels, $67.8 \%$ of patients were receiving diuretics, and $45.8 \%$ were taking $\beta$-blockers.

Almost all patients (94\%) had a medical history of hypertension. Neuropathy and retinopathy were the most common diabetic complications, with a prevalence of $20.0 \%$ and $19.9 \%$, respectively (table 3 ). In terms of CV history, with ARTS-DN excluding patients with symptomatic HFrEF, the most common disorder was myocardial ischemia (9.4\%; table 3 ).

Only $18.9 \%$ of patients were current smokers, whereas $41.8 \%$ were former smokers and $39.3 \%$ had never smoked. A large proportion of patients (49.9\%) did not drink alcohol, 39.8\% were light drinkers, 9.9\% were moderate drinkers and $3.0 \%$ were heavy drinkers. 


\section{Discussion}

Recent developments in DKD research, including the clinical evaluation of several new candidates for DKD therapy, such as bardoxolone, aliskiren and darbepoetin alfa, have failed to achieve the ultimate aim of limiting both progression to ESRD and CV morbidity/mortality in long-term studies in patients with DKD [29-31]. There is strong evidence that MRAs in combination with ACEIs or ARBs reduce mortality, morbidity, and rates of hospitalization in individuals with $\mathrm{HFrEF}[24,25,32]$. As a result, the steroidal MRAs eplerenone and spironolactone are recommended in international guidelines for the treatment of patients with HFrEF who remain symptomatic with ACEIs and $\beta$-blockers $[23,33,34]$. These MRAs have also shown promise in early studies in patients with DKD or CKD [17, 19-22, 35]. However, MRAs as a drug class are often underused, especially in patients with diabetes and/or renal dysfunction, because steroidal MRAs are associated with an increased risk of hyperkalemia [17, $28,36]$. There is thus an urgent need to evaluate novel therapies that might safely improve CV and renal outcomes in patients with DKD.

Finerenone shows higher selectivity for the MR over other steroid hormone receptors compared with spironolactone and improved affinity for the MR compared with eplerenone in vitro [26]. In preclinical studies, finerenone was seen to distribute equally to the heart and kidneys in rats [27]. Finerenone might therefore confer end-organ protection with a reduced risk of electrolyte perturbation compared with the marketed MRAs eplerenone and spironolactone. In ARTS, a phase 2a study of finerenone in patients with $\mathrm{HFrEF}$ and mild-to-moderate $\mathrm{CKD}$, finerenone decreased levels of albuminuria to at least the same degree as spironolactone. Furthermore, finerenone was associated with only infrequent and mostly mild adverse events and was associated with significantly smaller mean increases in serum potassium concentration and a lower incidence of both hyperkalemia and worsening renal function than spironolactone [28]. As hyperkalemia is a major limitation of present treatment strategies with MRAs, finerenone may significantly change the therapeutic possibilities in patients with CKD.

ARTS-DN is the first large clinical trial of finerenone in combination with a RAS inhibitor in patients with type 2 diabetes and a clinical diagnosis of $\mathrm{DN}$. Owing to the inclusion criteria, UACRs and levels of $\mathrm{HbA}_{1 \mathrm{c}}$ were elevated above the normal range in the study population. Average systolic blood pressure was also slightly higher

Finerenone for Type 2 Diabetes Mellitus and Diabetic Nephropathy than normal, as expected in patients with type 2 diabetes mellitus and DKD $[19,21]$; however, the average blood pressure reading was within the normal range. Despite the high prevalence of historical hypertension in the study population, and because of the exclusion of patients with severe hypertension from this study, patients generally had good blood pressure control at baseline. This may mean that only small changes in blood pressure can be expected under treatment with finerenone over 90 days in ARTS-DN.

Both type 2 diabetes mellitus and CKD are associated with an elevated risk of CV disease, and hence it is no surprise that a substantial proportion (39.6\%) of patients in ARTS-DN have a history of CV disorders. The percentage of patients using lipid-lowering agents in this study is in line with that seen in other studies of patients with type 2 diabetes mellitus who are at $\mathrm{CV}$-renal risk $[37,38]$. Previously, in ARTS, finerenone (10 mg once daily/5 mg twice daily) in combination with the standard of care for HFrEF was shown to be at least as effective as spironolactone at decreasing levels of hemodynamic stress biomarkers (BNP and NT-proBNP) as well as at reducing albuminuria. Even though patients with symptomatic HFrEF were excluded from ARTS-DN because a placebo-group would have been unethical given the knowledge that MRAs are life-prolonging in such patients, it is still predicted that a large number of participants in ARTS-DN, especially those with CV disease, might experience a dual CV-renal benefit from finerenone treatment. The 2013 American College of Cardiology HF guidelines state that clinical evaluations of patients with HF are best informed by the use of multiple biomarkers, including natriuretic peptides and indicators of both myocardial fibrosis and injury [34]. In ARTS-DN, the levels of galectin-3 (a marker of myocardial fibrosis) and troponin $\mathrm{T}$ (a marker of myocardial injury) will be monitored along with the levels of the natriuretic peptides BNP and NT-proBNP, which are differentially affected by renal function. Assessments of the levels of soluble ST-2, another marker of myocardial fibrosis, will not be performed in this phase $2 \mathrm{~b}$ study. Markers of renal function will, however, be followed and should provide further insight into cardiac health because renal injury is thought to be involved in the progression of HF [34].

Should finerenone satisfy the criteria of reducing albuminuria as well as being well tolerated and having a good safety profile in this phase $2 \mathrm{~b}$ trial, there are plans to initiate a comprehensive phase 3 program of clinical trials in patients with DKD. After the failure of other potential therapies such as bardoxolone, aliskiren and darbepoetin 
alfa to progress beyond the clinical trial stage of drug development, new medications in this field are keenly awaited. It is hoped that eventually finerenone will succeed in fulfilling the unmet clinical need of improving long-term cardiovascular and renal outcomes in patients with DKD.

\section{Conclusion}

ARTS-DN is a multicenter, randomized, double-blind, placebo-controlled, parallel-group, phase $2 \mathrm{~b}$ study started in June 2013. The recruitment rate was faster than expected, and enrolment had to be stopped more than 6 months earlier than planned. The trial was clinically completed in August 2014. Based on the results of this trial, future long-term clinical studies examining the effects of finerenone on the progression of renal disease as well as on CV morbidity and mortality in patients with DKD would be warranted [39].

\section{Conflicts of Interest}

R.A. has participated in Steering Committees for Abbvie, Bayer HealthCare AG and Sandoz; has performed data safety monitoring for Amgen and Celgene; has been a consultant to AstraZeneca, Daiichi Sankyo, Genkyotex, Eli Lilly and Takeda Pharma; and has received grant and research funding from VA and NIH. G.L.B. has received an investigator-initiated grant/research support from Takeda (direct funding to University of Chicago); has been a principal investigator in national/international clinical trials sponsored by Bayer HealthCare AG, Medtronic and Relypsa (direct funding to University of Chicago); and has been an advisor/consultant for AbbVie, Bayer HealthCare AG, BMS, CVRx, Elcelyx, Eli Lilly/ Boehringer-Ingelheim, Janssen, Medtronic, Novartis GSK, Takeda, Tengion and ZS Pharma. J.C.C. is a member of steering committees of international projects funded by AstraZeneca, Bayer HealthCare AG, Lilly, MSD, Pfizer and Sanofi; is a member of global/regional advisory boards of Boehringer-Ingelheim, Eli Lilly, MSD, Pfizer; and has received honorarium and travelling support from Amylin, AstraZeneca, Bayer HealthCare AG, Boehringer-Ingelheim, Bristol Myers Squibb, Daiichi-Sankyo, GlaxoSmithKline, Impeto, Lilly, MSD, Novartis, Pfizer, Sanofi and Takeda, with the honorarium donated to the Chinese University of Hong Kong for diabetes research and education; her institution has also received research and educational grants from these companies. M.E.C. has received grants and lecture fees from Novo-Nordisk, AbbVie, BoehringerIngelheim, Eli Lilly, Bayer HealthCare AG, MSD and AstraZeneca. A.C.F., N.K.K. and C.N. are employees of Bayer HealthCare AG. R.T.G. has consultancy agreements with AbbVie, Bayer HealthCare AG, Ipsen, Novartis Pharma and Otsuka Pharmaceuticals - no personal remuneration is accepted (compensations are paid to his institution for research). H.H. and R.S. have received honoraria for presentations from Bayer HealthCare AG. A.P. provided clinical trial support funded by Bayer HealthCare AG. G.R. has consultancy agreements with Alexion Pharmaceuticals, Bayer HealthCare AG, Novartis Pharma, and REATA Pharmaceuticals - no personal remuneration is accepted (compensations are paid to his institution for research and educational activities). P.R. has consultancy agreements with AbbVie, AstraZeneca, Bayer HealthCare AG, BMS, Eli Lilly, Boehringer Ingelheim, Novartis Pharma, and Novo Nordisk: no personal remuneration is accepted (compensations are paid to his institution). L.M.R. has been a speaker and advisor for Bayer HealthCare AG.

\section{Funding}

Bayer HealthCare AG provided financial support for the conduct of the research and preparation of the article. They were involved in the study design, collection, analysis, and interpretation of baseline data, the writing of the report and the decision to submit the article for publication. Dr Lucy Bomphrey of Oxford PharmaGenesis ${ }^{\mathrm{TM}}$ provided medical writing support funded by Bayer HealthCare AG.

\section{References}

1 Collins AJ, Foley RN, Chavers B, Gilbertson D, Herzog C, et al: US Renal Data System 2013 Annual Data Report. Am J Kidney Dis 2014;63:A7.

-2 Bakris GL, Molitch M: Microalbuminuria as a risk predictor in diabetes: the continuing saga. Diabetes Care 2014;37:867-875.

-3 Parving HH, Lewis JB, Ravid M, Remuzzi G, Hunsicker LG: Prevalence and risk factors for microalbuminuria in a referred cohort of type II diabetic patients: a global perspective. Kidney Int 2006;69:2057-2063.

4 de Jong PE, Gansevoort RT: Albuminuria in non-primary renal disease: risk marker rather than risk factor. Nephrol Dial Transplant 2010;25:656-658.

5 Schmieder RE, Mann JF, Schumacher H, Gao $\mathrm{P}$, Mancia $\mathrm{G}$, et al: Changes in albuminuria predict mortality and morbidity in patients with vascular disease. J Am Soc Nephrol 2011; 22:1353-1364.

6 de Zeeuw D, Remuzzi G, Parving HH, Keane WF, Zhang Z, et al: Proteinuria, a target for renoprotection in patients with type 2 diabetic nephropathy: lessons from RENAAL. Kidney Int 2004;65:2309-2320.

7 Schmieder RE, Schutte R, Schumacher $\mathrm{H}$, Bohm M, Mancia G, et al: Mortality and morbidity in relation to changes in albuminuria, glucose status and systolic blood pressure: an analysis of the ONTARGET and TRANSCEND studies. Diabetologia 2014;57:2019-2029.

8 American Diabetes Association: Standards of medical care in diabetes - 2014. Diabetes Care 2014;37(suppl 1):S14-S80.
9 Schmieder RE, Ruilope LM, Barnett AH: Renal protection with angiotensin receptor blockers: where do we stand. J Nephrol 2011; 24:569-580.

10 Kalaitzidis R, Bakris GL: Effects of angiotensin II receptor blockers on diabetic nephropathy. J Hypertens Suppl 2009;27:S15S21.

11 American Diabetes Association: Executive summary: standards of medical care in diabetes - 2014. Diabetes Care 2014;37(suppl 1):S5S13.

12 Tang WH, Vagelos RH, Yee YG, Benedict CR Willson K, et al: Neurohormonal and clinical responses to high- versus low-dose enalapril therapy in chronic heart failure. J Am Coll Cardiol 2002;39:70-78. 


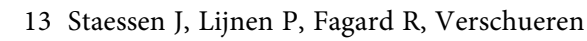
LJ, Amery A: Rise in plasma concentration of aldosterone during long-term angiotensin II suppression. J Endocrinol 1981;91:457465.

14 Bomback AS, Klemmer PJ: The incidence and implications of aldosterone breakthrough. Nat Clin Pract Nephrol 2007;3:486-492.

$\checkmark 15$ Schjoedt KJ, Andersen S, Rossing P, Tarnow $\mathrm{L}$, Parving $\mathrm{HH}$ : Aldosterone escape during blockade of the renin-angiotensin-aldosterone system in diabetic nephropathy is associated with enhanced decline in glomerular filtration rate. Diabetologia 2004;47:19361939.

16 Horita Y, Taura K, Taguchi T, Furusu A, Kohno S: Aldosterone breakthrough during therapy with angiotensin-converting enzyme inhibitors and angiotensin II receptor blockers in proteinuric patients with immunoglobulin A nephropathy. Nephrology (Carlton) 2006; 11:462-466.

17 Navaneethan SD, Nigwekar SU, Sehgal AR, Strippoli GF: Aldosterone antagonists for preventing the progression of chronic kidney disease. Cochrane Database Syst Rev 2009; 3:CD007004

18 Bomback AS, Kshirsagar AV, Amamoo MA, Klemmer PJ: Change in proteinuria after adding aldosterone blockers to ACE inhibitors or angiotensin receptor blockers in CKD: a systematic review. Am J Kidney Dis 2008;51: 199-211.

19 Chrysostomou A, Becker G: Spironolactone in addition to ACE inhibition to reduce proteinuria in patients with chronic renal disease. N Engl J Med 2001;345:925-926.

20 Rossing K, Schjoedt KJ, Smidt UM, Boomsma F, Parving HH: Beneficial effects of adding spironolactone to recommended antihypertensive treatment in diabetic nephropathy: a randomized, double-masked, cross-over study. Diabetes Care 2005;28:2106-2112.

-21 Sato A, Hayashi K, Naruse M, Saruta T: Effectiveness of aldosterone blockade in patients with diabetic nephropathy. Hypertension 2003;41:64-68.
22 Epstein M, Williams GH, Weinberger M, Lewin A, Krause S, et al: Selective aldosterone blockade with eplerenone reduces albuminuria in patients with type 2 diabetes. Clin J Am Soc Nephrol 2006;1:940-951.

23 McMurray JJ, Adamopoulos S, Anker SD, Auricchio A, Bohm M, et al: ESC Guidelines for the diagnosis and treatment of acute and chronic heart failure 2012: The Task Force for the Diagnosis and Treatment of Acute and Chronic Heart Failure 2012 of the European Society of Cardiology. Developed in collaboration with the Heart Failure Association (HFA) of the ESC. Eur Heart J 2012;33:1787-1847.

24 Pitt B, Zannad F, Remme WJ, Cody R, Castaigne A, et al: The effect of spironolactone on morbidity and mortality in patients with severe heart failure. Randomized Aldactone Evaluation Study Investigators. N Engl J Med 1999;341:709-717.

25 Zannad F, McMurray JJ, Krum H, van Veldhuisen DJ, Swedberg K, et al: Eplerenone in patients with systolic heart failure and mild symptoms. N Engl J Med 2011;364:11-21.

26 Bärfacker L, Kuhl A, Hillisch A, Grosser R, Figueroa-Perez S, et al: Discovery of BAY 94 8862: a nonsteroidal antagonist of the mineralocorticoid receptor for the treatment of cardiorenal diseases. ChemMedChem 2012;7: 1385-1403.

27 Kolkhof P, Delbeck M, Kretschmer A, Steinke W, Hartmann E, et al: Finerenone, a novel selective nonsteroidal mineralocorticoid receptor antagonist protects from rat cardiorenal injury. J Cardiovasc Pharmacol 2014;64:6978.

28 Pitt B, Kober L, Ponikowski P, Gheorghiade M, Filippatos G, et al: Safety and tolerability of the novel non-steroidal mineralocorticoid receptor antagonist BAY 94-8862 in patients with chronic heart failure and mild or moderate chronic kidney disease: a randomized, double-blind trial. Eur Heart J 2013;34:24532463.

29 Parving HH, Brenner BM, McMurray JJ, de Zeeuw D, Haffner SM, et al: Cardiorenal end points in a trial of aliskiren for type 2 diabetes. N Engl J Med 2012;367:2204-2213.

-30 Pfeffer MA, Burdmann EA, Chen CY, Cooper ME, de Zeeuw D, et al: A trial of darbepoetin alfa in type 2 diabetes and chronic kidney disease. N Engl J Med 2009;361:2019-2032. de Zeeuw D, Akizawa T, Audhya P, Bakris GL, Chin M, et al: Bardoxolone methyl in type 2 diabetes and stage 4 chronic kidney disease. $\mathrm{N}$ Engl J Med 2013;369:2492-2503.

- 32 Pitt B, Remme W, Zannad F, Neaton J, Martinez F, et al: Eplerenone, a selective aldosterone blocker, in patients with left ventricular dysfunction after myocardial infarction. N Engl J Med 2003;348:1309-1321.

33 Butler J, Ezekowitz JA, Collins SP, Givertz MM, Teerlink JR, et al: Update on aldosterone antagonists use in heart failure with reduced left ventricular ejection fraction. Heart Failure Society of America Guidelines Committee. J Card Fail 2012;18:265-281.

34 Yancy CW, Jessup M, Bozkurt B, Butler J, Casey DE Jr, et al: 2013 ACCF/AHA guideline for the management of heart failure: a report of the American College of Cardiology Foundation/American Heart Association Task Force on Practice Guidelines. J Am Coll Cardiol 2013;62:e147-e239.

-35 Toto RD: Aldosterone blockade in chronic kidney disease: can it improve outcome? Curr Opin Nephrol Hypertens 2010;19:444-449.

36 Pitt B, Filippatos G, Gheorghiade M, Kober L, Krum H, et al: Rationale and design of ARTS: a randomized, double-blind study of BAY 948862 in patients with chronic heart failure and mild or moderate chronic kidney disease. Eur J Heart Fail 2012;14:668-675.

37 Lambers Heerspink HJ, Chertow GM, Akizawa T, Audhya P, Bakris GL, et al: Baseline characteristics in the Bardoxolone methyl EvAluation in patients with Chronic kidney disease and type 2 diabetes mellitus: the Occurrence of renal eveNts (BEACON) trial. Nephrol Dial Transplant 2013;28:2841-2850.

-38 Parving HH, Brenner BM, McMurray JJ, de Zeeuw D, Haffner SM, et al: Baseline characteristics in the Aliskiren Trial in Type 2 Diabetes Using Cardio-Renal Endpoints (ALTITUDE). J Renin Angiotensin Aldosterone Syst 2012;13:387-393.

39 Ruggenenti P, Cravedi P, Remuzzi G: The RAAS in the pathogenesis and treatment of diabetic nephropathy. Nat Rev Nephrol 2010; 6:319-330.
Finerenone for Type 2 Diabetes Mellitus and Diabetic Nephropathy
Am J Nephrol 2014;40:572-581 DOI: $10.1159 / 000371497$ 